\title{
Growth and characterization of L-Arginine Potassium Iodide (LAPI) Semi organic single crystals
}

\author{
G. $\operatorname{Kanagan}^{1}$, S. Pari ${ }^{2 *}$ \\ ${ }^{1,2}$ Department of Physics, National College (Autonomous), Triuchirappalli-620 001
}

Available online at: www.isroset.org

Received: 19/May/2019, Accepted: 06/Jun/2019, Online: 30/Jun/2019

\begin{abstract}
A novel semiorganic NLO material viz., L-arginine potassium iodide (LAPI) has been synthesized by slow evaporation technique. The crystal structure and lattice parameters of the grown LAPI crystals have been determined using single crystal X-ray diffraction and powder X-ray diffraction techniques. Single crystal X-ray diffraction study shows that the material crystallizes in monoclinic structure with the non-centrosymmetric space group P2 $2_{1}$. The FTIR spectrum was used to identify the various functional groups and modes of vibrations present in LAPI crystal. The mechanical strength of the crystal is estimated by Vicker's hardness test and the obtained results are discussed.
\end{abstract}

Keywords- Solution growth, L-arginine complex, XRD, FTIR, UV

\section{INTRODUCTION}

Semiorganic compound crystals have a lot of non-linear optical (NLO) applications like terahertz wave generation, frequency conversion, electro-optic light modulation, optical computing, optical communication and deflectors in optically operated fibres. The semiorganic single crystals have the properties of both organic and inorganic materials. Many amino acids contain protonated amino group and a deprotonated carboxylic group [1-3]. There are more than 20 genetically encoded amino acids found in proteins. The crystal structure of arginine has not been completely solved. From the literature survey, it is noticed that the crystal structures of L-arginine dihydrate, DL-arginine monohydrate and DL-arginine dihydrate have been reported [4-7]. A semiorganic NLO crystal viz., L-arginine hydrochloride has been grown and the physical properties such as thermal expansion, elastic properties and dielectric characteristics were studied $[8,9]$.

For instance, Sudeshna Mukerji et al [8,9] have grown the crystals of L-arginine hydrochloride and L-arginine hydrobromide and the hardness properties were found. Knoop microhardness anisotropy on the cleavage plane (100) of single crystals of L-arginine hydrochloride and L-arginine hydrobromide is reported. The microhardness increases from (001) to (011) and then decreases to (010) orientation. Calculation of Young's modulus values for (100), (010), and (001) planes of the sample were done [10]. In this work, single crystals of L-arginine potassium iodide were grown by solution method and the grown crystals were subjected to various studies like XRD studies, UV-visible spectral studies, hardness studies, dielectric studies, SHG studies etc and the obtained results were analyzed and reported in this paper.

\section{SYNTHESIS AND CRYSTAL GROWTH}

L-arginine potassium iodide (LAPI) salt was synthesized from stoichiometric incorporation of AR grade of L-arginine and potassium iodide in 1:1 molar ratio. The reactant salts were dissolved in double distilled water and mixed thoroughly using a magnetic stirrer at $40^{\circ} \mathrm{C}$ for about 3 hours. Here there was a slight evaporation of water during the reaction. On heating the solution at $50^{\circ} \mathrm{C}$ for about 6 hours, there was further evaporation of the solution and the synthesized salt of LAPI was obtained. Single crystals of LAPI were grown by solution method with slow evaporation technique using water as the solvent. The saturated solution of the synthesized salt of LAPI was prepared by dissolving the salt in double distilled water by continuous stirring of the solution using a magnetic stirrer and the saturated solution was filtered using $4 \mu \mathrm{m}$ Whatman filter paper. As the solution evaporates slowly, the saturated solution was changed into supersaturated solution and crystal nuclei are formed in the 
solution. On further evaporation of solution, crystal nuclei were grown into big-sized crystals and bulk crystals of LAPI were obtained after the growth period of about 30 days. The photograph of harvested crystal of LAPI is shown in the Figure 1.

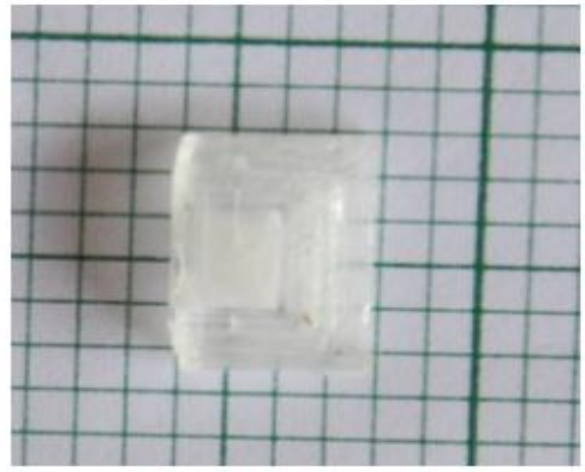

Fig.1: The grown crystal of LAPI

\section{III.RESULT AND DISCUSSION}

\subsection{Single crystal XRD studies}

Single crystal XRD data have been obtained using an ENRAF NONIUS CAD4-MV31 single crystal X-ray diffractometer with $\operatorname{MoK}_{\alpha}(\lambda=0.71073 \AA)$. Here a good quality crystal of LAPI of dimensions of $0.4 \times 0.4 \times 0.3 \mathrm{~mm}^{3}$ was used. This study is used to find the crystal structure and lattice parameters and the principle used in this experiment is the Bragg's law. The obtained single crystal XRD data for the grown crystal of LAPI are provided in the table1. From the data, it is noticed that LAPI crystal crystallizes in monoclinic structure. The morphology of the grown crystal of LAPI was analysed by winxmorph software program and the indexed morphology of LAPI is shown in the Figure 2.

Table 1: Single crystal XRD data for LAPI crystal

\begin{tabular}{|c|c|}
\hline 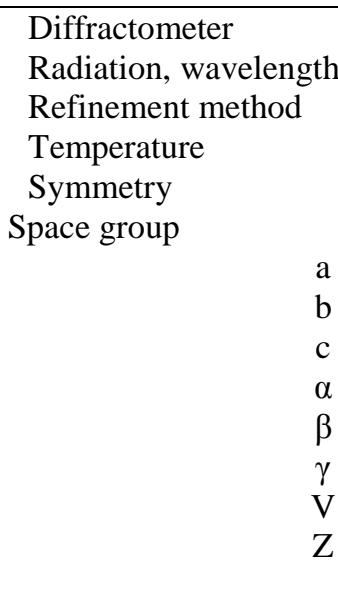 & $\begin{array}{l}\text { ENRAF NONIUS CAD- } 4 \\
\text { MoK } \\
0.71069 \AA \\
\text { Full matrix Least square method } \\
293(2) \mathrm{K} \\
\text { Monoclinic } \\
\mathrm{P} 2_{1} \\
6.157(3) \AA \\
8.284(4) \AA \\
10.946(2) \AA \\
90^{\circ} \\
98.25(3)^{\circ} \\
90^{\circ} \\
552.51(4) \AA^{3} \\
2\end{array}$ \\
\hline
\end{tabular}

\subsection{Powder XRD studies}

The powder X-ray diffraction (PXRD) study was carried out for the powder form of the grown LAPI crystal to ascertain the crystal structure and to identify the diffraction planes. The PXRD pattern of the sample is shown in Figure 3 and the welldefined peaks at specific $2 \theta$ values show high crystalline of the grown crystal. The diffraction planes of the powder XRD pattern were indexed using the INDEXING software package. The unit cell parameters of the grown LAPI crystals were found 
using the UNITCELL software package and these values are observed to be coinciding with the data obtained from single crystal XRD studies.

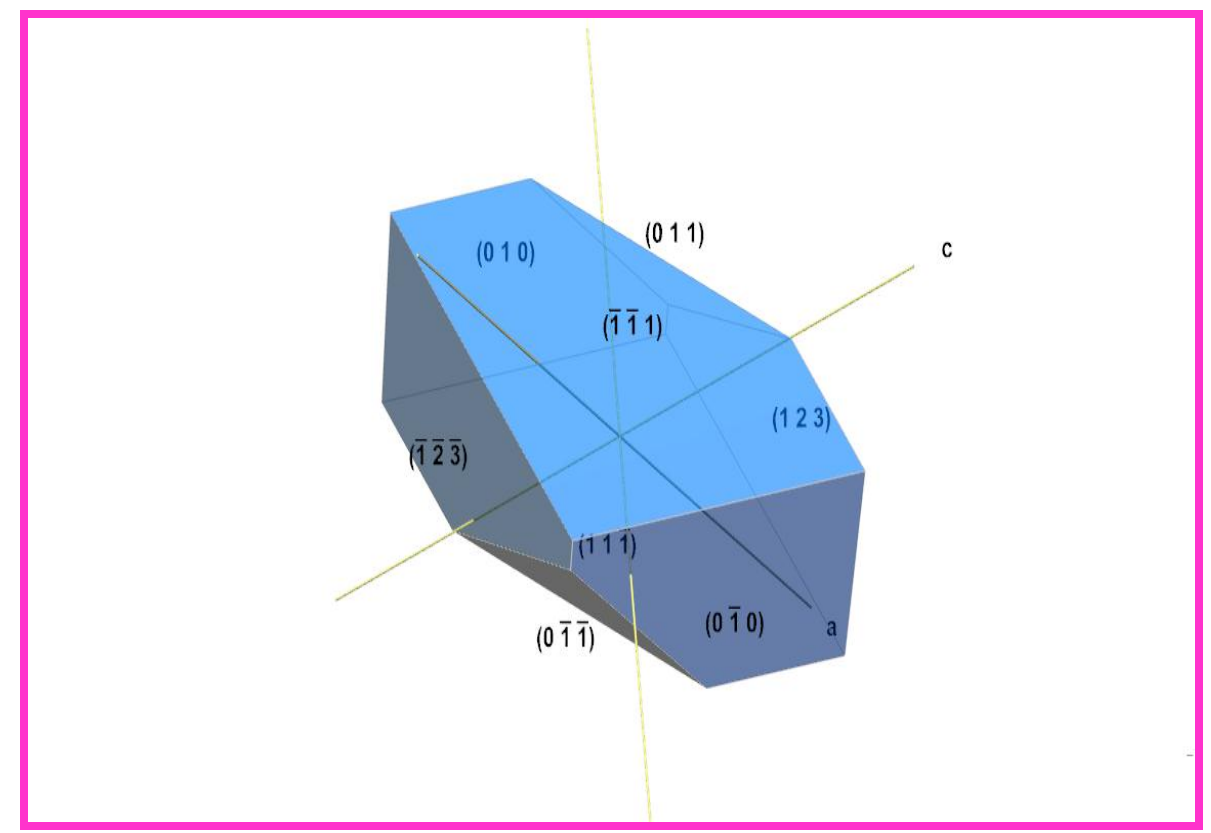

Fig.2: Indexed morphology of LAPI crystal

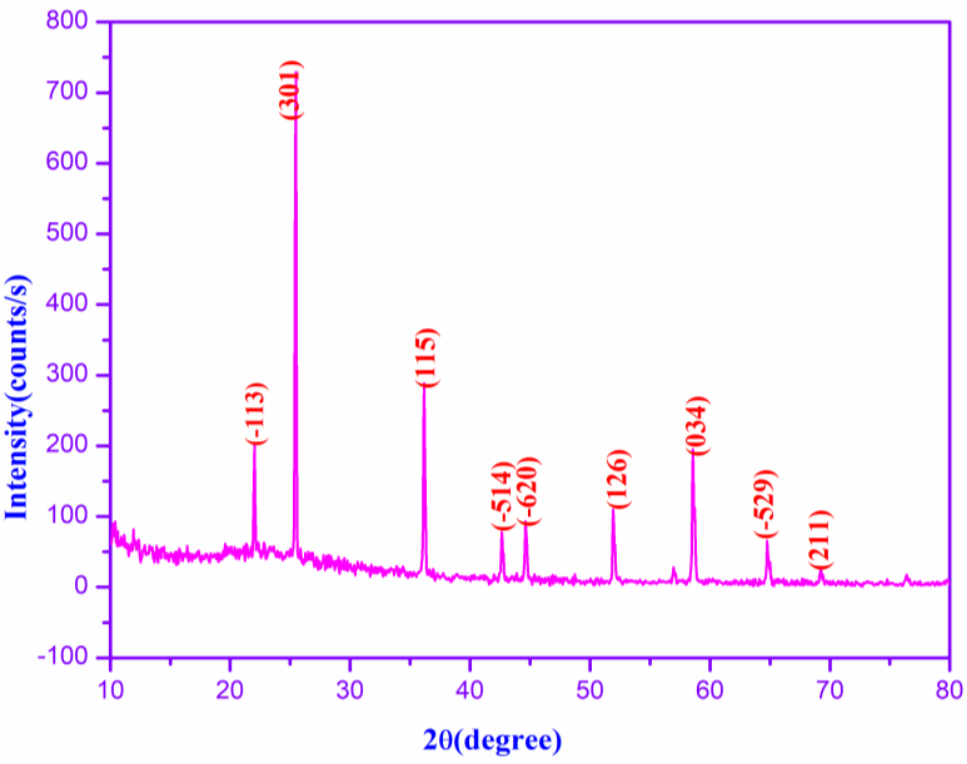

\subsection{FTIR studies}

Fig. 3: Powder XRD spectrum of LAPI crystal

The presence of functional groups, absorption peaks and the nature of the bonds present in the title material were assessed by the FTIR spectral analysis using PERKIN ELMER FTIR spectrophotometer with $\mathrm{KBr}$ technique for the range 4000-400 $\mathrm{cm}^{-1}$. The recorded FTIR spectrum of LAPI crystal is shown in the Figure 4. The peak observed at $1396 \mathrm{~cm}^{-1}$ is assigned to $\mathrm{CH}_{2}$ bending. The peak at $1625 \mathrm{~cm}^{-1}$ is corresponding to the combination of both $\mathrm{NH}_{3}{ }^{+}$bending and $\mathrm{COO}^{-}$ stretching vibration. The C-H stretch is noted at $2927 \mathrm{~cm}^{-1}$. The band at $3392 \mathrm{~cm}^{-1}$ is due to $\mathrm{NH}_{3}{ }^{+}$stretching vibration. The peak at $1111 \mathrm{~cm}^{-1}$ is assigned to scissoring mode of $\mathrm{COO}^{-}$and the peak at $603 \mathrm{~cm}^{-1}$ can be considered to be vibration of K-I bond. The various functional groups of LAPI and their assignments are presented in the table 2. Here the assignments to the vibration peaks/bands are given in the accordance with the data reported in the literature [11,12]. 


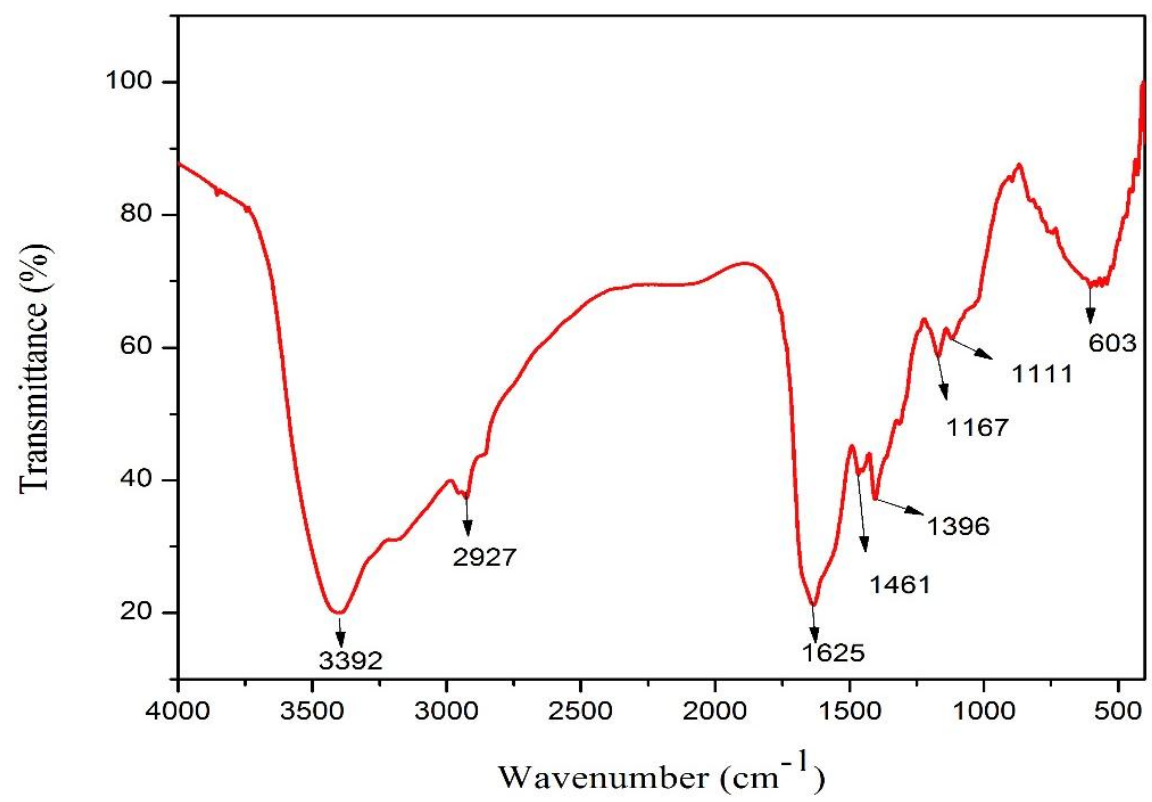

Fig.4: FTIR spectrum of LAPI crystal

Table 2: FTIR spectral assignments for LAPI crystal

\begin{tabular}{|l|l|}
\hline Wave number $\left(\mathbf{c m}^{-1}\right)$ & Band/peak assignments \\
\hline 3392 & $\mathrm{NH}_{3}{ }^{+}$stretching vibration \\
\hline 2927 & $\mathrm{C}-\mathrm{H}$ stretching \\
\hline 1625 & $\begin{array}{l}\mathrm{NH}_{3}{ }^{+} \text {bending and } \mathrm{COO}^{-} \\
\text {stretching mode }\end{array}$ \\
\hline 1461 & $\mathrm{CH}_{2}$ bending \\
\hline 1396 & $\mathrm{CH}_{2}$ bending \\
\hline 1167 & $\mathrm{C}-\mathrm{O}$ stretching \\
\hline 1111 & Scissoring mode of $\mathrm{COO}^{-}$ \\
\hline 603 & K-I mode \\
\hline
\end{tabular}

\subsection{Microhardness studies}

The Vickers microhardness values were estimated by using the SHIMADZU HMV microhardness tester by applying different loads. The microhardness analysis was carried out using the polished plane of the crystal. The hardness number was calculated using the relation

$$
\mathrm{H}_{\mathrm{V}}=1.8544\left(\mathrm{P} / \mathrm{d}^{2}\right) \mathrm{kg} / \mathrm{mm}^{2}
$$

where $\mathrm{d}$ is the average diagonal length of the indentation $[13,14]$ and $\mathrm{P}$ is the applied load. Figure.5 shows plot of the hardness number and applied load. It is seen that the microhardness number increases with increasing of load and this indicates reverse indentation size effect. The relationship between load and indentation diagonal length is given by well known Meyer's law $\mathrm{P}=\mathrm{K}_{1} \mathrm{~d}^{\mathrm{n}}$, where $\mathrm{k}_{1}$ is a material constant and $\mathrm{n}$ are the Meyer's index. The value of Meyer's index was calculated by drawing the plot of $\log \mathrm{d}$ versus $\log \mathrm{P}$ which is shown in The Figure 6. The obtained value of Meyer's index or work hardening coefficient of LAPI crystal is 3.402. Since this value is more than 1.6, LAPI crystal is a soft material. 


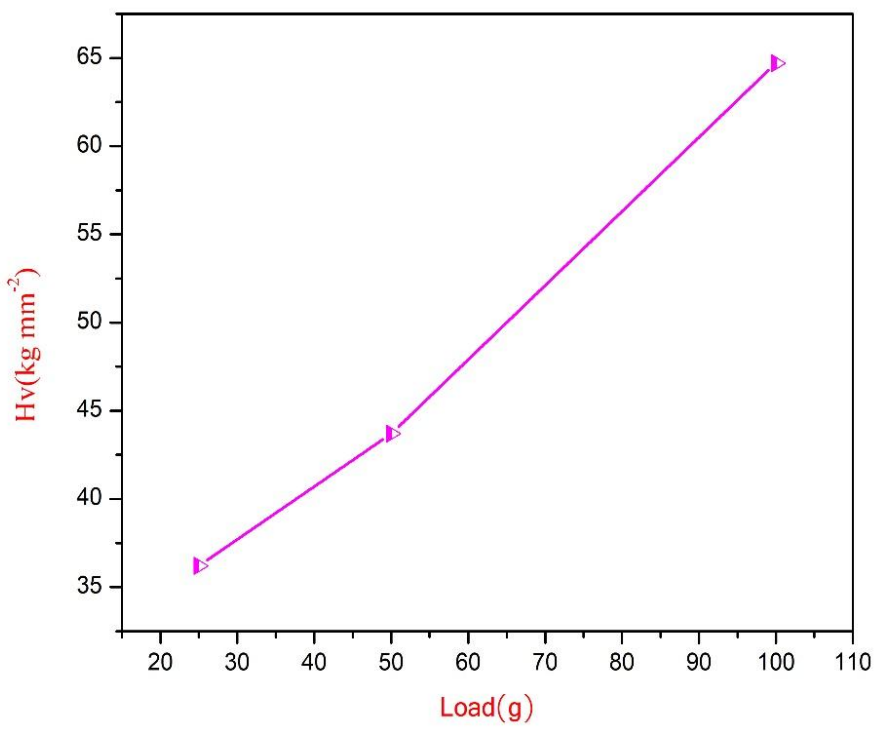

Fig.5: Variation of hardness with load for LAPI crystal

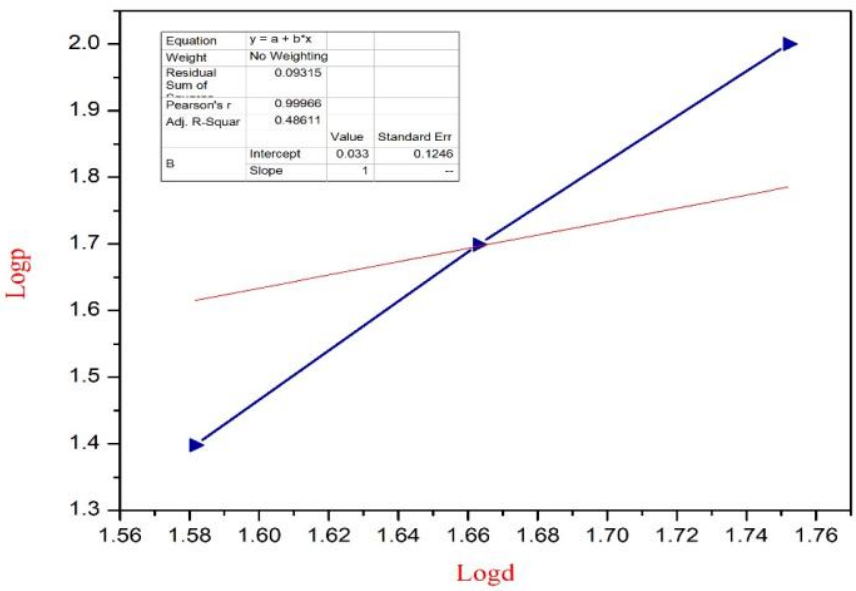

Fig.6: Plot of $\log$ P versus $\log \mathrm{d}$ for LAPI crystal

\section{CONCLUSION}

Semiorganic crystals of L-arginine potassium iodide (LAPI) were grown successfully by a slow evaporation method. XRD study confirms the monoclinic crystal system of the sample with a non-centerosymmetric space group. The functional groups of LAPI crystal were found. The peak observed at $1396 \mathrm{~cm}^{-1}$ is assigned to $\mathrm{CH}_{2}$ bending whereas the peak at $1625 \mathrm{~cm}^{-1}$ is corresponding to the combination of both $\mathrm{NH}_{3}{ }^{+}$bending and $\mathrm{COO}^{-}$stretching vibration. We have also tabulated the various functional group associated with LAPI crystal. The hardness of the sample was found to be high and it increases with increase of applied load. Such results indicate a reverse indentation size effect. This work paves a way to synthesis other such semiorganic crystal by a simple growth method.

\section{ACKNOWLEDGMENT}


The authors are thankful to ACIC, St. Joseph's college (Trichy) and STIC, Cochin University, Cochin and Sastra University Thanjavur, SAIF, IIT Madras for providing the facilities taken all the studies and carried out the research work successfully.

\section{REFERENCES}

[1]K.Ambujam,K.Rajarajan,S.Selvakumar,J.Madhavan,GulamMohamed,P.Sagayaraj,Opt.er., 29 (2007) 657.

[2] K. Selvaraju, R. Valluvan, S. Kumararaman Mater. Lett., 60 (2006) 2848.

[3] R. Shanmugavadivu, G.Ravi, A.Nixon Azariah J.Phys.Chem.Solids 67 (2006) 1858.

[4]S.Skathi Priya , A. Alexandar, P. Surendran,A.Lakshmanan ,P.Rameshkumar,P.Sagayaraj, Optical Materials 66 (2017) 434-441

[5] P. Anandan , T. Saravanan, G. Parthipan, R. Mohan Kumar, G. Bhagavannarayana ,G. Ravi , R. Jayavel, Solid State Sciences 13 (2011) 915 922

[6] P. Anandan, G. Parthipan, K. Pazhanivel, G. Ravi, R. Jayavel, Optik 125 (2014) 8-10

[7] S. Arjunan, A. Bhaskaran, R. Mohan Kumar, R. Mohan, R. Jayavel, Journal of Alloys and Compounds 506 (2010) $784-787$.

[8] S. Hausshe, J. Chrosch, F. Gnanam, E. Fiorentini, K. Recker, F. Wallrafen, Cryst. Res. Technol. 25, 617 (1990).

[9] S. Mukerji, T. Kar, Mater. Chem. Phys.57, 72 (1998).

[10] Sudeshna Mukerji, Tanusree Kar, Materials Research Bulletin 35 (2000) 711-717.

[11] P.Kalsi, Spectroscopy of Organic Compounds, Wiley Eastern, New Delhi, 1985.

[12] J. Packiam Julius, S.A. Rajasekar, A. Joseph Arul Pragasam, V. Joseph, P. Sagayaraj,Materials Science and Engineering B107 (2004) $259-263$.

[13] A.S. Haja Hameed, G. Ravi, R. Dhanasekaran, P. Ramasamy, J. CrystGrowth,212(2000) 227.

[14] J.N. Sherwood, pure. Appl. Opt. 7 (1998) 229. 